\title{
Perfil microbiológico bucal dos pacientes portadores de osteonecrose maxilar induzida por bisfosfonatos
}

\author{
Oral microbiological profile from bisphosphonate-related osteonecrosis of the jaw patients
}

Brenna Karoline Sousa Lima ${ }^{1}$, Simeone Júlio dos Santos Castelo Branco ${ }^{2,4}$, Valéria Costa Fontes ${ }^{2,}$ ${ }^{4}$, Aruanã Joaquim Matheus Costa Rodrigues Pinheiro ${ }^{4}$, Viviane Hass ${ }^{1,3}$, Lidio Gonçalves Lima Neto $^{4}$, Patrícia Maria Wiziack Zago ${ }^{1}$

\begin{abstract}
Resumo: A osteonecrose maxilar induzida por bisfosfonatos (OMIB) é uma lesão necrótica dos ossos maxilares associada à utilização de medicações antirreabsortivas como os bisfosfonatos. A patogênese da OMIB ainda não está bem estabelecida, o que dificulta a sua prevenção, prognóstico e tratamento clínico. Diversos são os fatores de risco associados ao desenvolvimento da lesão, dentre eles, a presença de processos infecciosos no ambiente bucal. A fim de se verificar uma possível correlação entre a presença de microrganismos específicos e a patogênese e/ou progressão da OMIB, realizou-se esta revisão bibliográfica. Para isso, artigos científicos datados de 2003 a 2015 e que relacionassem os descritores "osteonecrosis", "jaw" e "micorganisms" foram pesquisados através da base de dados do Medline-Pubmed. Selecionaram-se publicações que relataram OMIB em humanos e que avaliaram o perfil microbiológico bucal desses pacientes. A maioria dos trabalhos analisados indicou que o gênero Actinomyces spp foi o mais prevalente em lesões de OMIB; no entanto, estudos que utilizaram técnicas de identificação biomolecular ressaltaram a presença de bactérias pertencentes ao Filo Firmicutes (Pseudoramibacter spp, Streptococcus spp, Parvimonas spp, Mogibacterium spp, Gemella spp, Eubacterium spp) e ao Filo Actinobacteria (Atopobium spp). Verifica-se que processos infecciosos participam ativamente da OMIB; portanto, a definição das espécies de microrganismos associados à patologia é essencial, tanto para o estabelecimento de uma adequada abordagem antimicrobiana quanto para a elucidação de sua patogenia. Nesse sentido, mais estudos são necessários para a determinação qualitativa e quantitativa do perfil microbiológico oral de pacientes com OMIB.
\end{abstract}

Palavras-chave: Bisfosfonatos. Osteonecrose. Bacteria. Microbiota.

\begin{abstract}
The bisphosphonate-related osteonecrosis of the jaw (BRONJ) is a necrotic lesion of the jaws associated with antiresorptive medications use, such as bisphosphonates. The BRONJ pathogenesis' still is not well established, which causes inadequate prevention, prognosis or medical treatment. There are several risk factors associated with the development of the injury, including the presence of infectious diseases in the oral environment. In order to verify a possible correlation between the presence of specific microorganisms and the pathogenesis and/or progression of BRONJ, a literature review was conducted. For this, scientific articles dated from 2003 to 2015 and that related descriptors as "osteonecrosis", "jaw" and "microorganisms" were searched through Medline-Pubmed database. Publications reporting an evaluation of the oral microbiological profile from BRONJ patients were selected. The majority of the studies indicated Actinomyces spp as the most prevalent bacteria genera found in BRONJ lesions. Therefore, researches that have used molecular microbiological identification techniques highlighted the presence of bacteria from Firmicutes phylum (Pseudoramibacter spp, Streptococcus spp, Parvimonas spp, Mogibacterium spp, Gemella spp, Eubacterium spp) and Actinobacteria phylum (Atopobium spp). Moreover, there is an active participation of infectious processes in BRONJ development and the elucidation of the microorganisms species' associated with that is an important measure even in an attempt to establish an adequate antimicrobial therapy or its pathogenesis. In this sense, further studies are required for the elucidation of the quantitative and qualitative oral microbiological profile from BRONJ patients.
\end{abstract}

Keywords: Bisphosphonate. Osteonecrosis. Bacteria. Oral microbiot

\footnotetext{
${ }^{1}$ Universidade CEUMA, Odontologia (Maranhão, Brasil)

${ }^{2}$ Universidade CEUMA, Biomedicina (Maranhão, Brasil)

${ }^{3}$ Universidade CEUMA, Programa de Pós-graduação em Odontologia (Maranhão, Brasil)

${ }^{4}$ Universidade CEUMA, Laboratório de Imunologia e Microbiologia de Infecções Respiratórias (Maranhão, Brasil)
}

Autor correspondente: Profa. Dra. Patrícia Maria Wiziack Zago. Programa de Graduação em Odontologia. Rua Josué Montello, 1, Renascença II. CEP: 65075120, São Luís, MA, Brasil. Tel.: (98) 32141427 


\section{Introdução}

Os bisfosfonatos são fármacos análogos sintéticos dos reguladores fisiológicos da calcificação e reabsorção óssea, os pirofosfatos ${ }^{1}$. A década de 60 data a descoberta e utilização dessas medicações para o tratamento de enfermidades ósseas que compreendem: a osteoporose, a doença de Paget, doenças de controle do metabolismo do cálcio e o emprego como adjuvante terapêutico em metástases ósseas e mieloma múltiplo ${ }^{2}$. Dentre as ações dos bisfosfonatos tem-se a inibição da atividade osteoclástica e consequentemente a inibição de mecanismos relacionados à reabsorção óssea, promovendo assim, os processos de formação desse tecido. No entanto, apesar de seus benefícios, uma importante complicação denominada osteonecrose maxilar induzida por bisfosfonatos (OMIB) tem sido relatada em pacientes usuários crônicos desses medicamentos ${ }^{2}$.

A OMIB foi primeiramente reconhecida em $2003^{3}$, quando houve 0 relato de 36 casos de pacientes com osteonecrose avascular dos maxilares associada à utilização de pamidronato ou zoledronato que, quimicamente, são classificados como compostos nitrogenados potentes utilizados por via intravenosa (IV). Bisfosfonatos utilizados por via oral (VO) como o alendronato ou risendronato (nitrogenados) ou os nãonitrogenados como etidronato (VO) e clodronato (VO e IV) apresentam menor potência ${ }^{4}$.

Desde o primeiro relato, acreditavase que somente os bisfosfonatos pudessem induzir à osteonecrose maxilar, porém, casos dessa patologia também foram relacionados à utilização de outras medicações antirreabsortivas, como os inibidores do ligante RANK e fármacos antiangiogênicos. A partir disso, e associado ao crescente número de novos casos de ostenecrose maxilar induzida por esses medicamentos, a
AAOMS (Associação Americana dos Cirurgiões Buco-Maxilo-Faciais, 2014) sugeriu a terminologia Osteonecrose Maxilar induzida por Medicamentos (OMIM) em substituição ao termo $\mathrm{OMIB}^{5}$.

Assim, a $\mathrm{AAOMS}^{2}$ define que pacientes podem ser considerados com OMIM se apresentarem as seguintes características: tratamento prévio ou atual com agentes antirreabsortivos ou antiangiogênicos; osso exposto ou osso que pode ser sondado através de uma fístula intra-oral ou extra-oral na região maxilo-facial com persistência de mais de oito semanas e ausência de histórico de terapia por radiação ou doença metastática evidente na maxila ${ }^{5}$.

Clinicamente, há uma exposição óssea de ocorrência espontânea ou evidenciada após procedimento odontológico como extração dentária, cirurgia periodontal, apicectomia e cirurgia de implantes dentários ${ }^{6,7}$. A área pode apresentar-se inchada, com sangramento e dolorida, $o$ que compromete a alimentação, a fala e a higiene oral do paciente. A ocorrência de ulceração é frequente e o osso necrótico exposto apresenta coloração brancoamarelada. Essas lesões podem permanecer assintomáticas por tempo indeterminado até o momento em que os tecidos locais apresentem inflamação ou evidência clínica de infecção. Os achados radiográficos são inespecíficos e assim, muitas vezes, é necessário biópsia para a determinação do diagnóstico ${ }^{9,10}$.

Por fim, não existe um tratamento comprovadamente eficaz para as lesões de OMIM, sendo que as principais medidas adotadas são a limpeza local, remoção de sequestro ósseo e administração de antibióticos locais e sistêmicos, visto que a infecção está frequentemente associada às lesões ósseas ${ }^{11}$.

A patogênese da OMIM ainda não está totalmente elucidada e muitas hipóteses têm sido estudadas. No entanto, sabe-se e que algumas 
condições são fatores de risco para o seu desenvolvimento, como a utilização de corticosteroides, o tipo da doença maligna, a realização de extrações dentárias e a presença de infecção local $^{5,10}$. Pesquisas têm sido realizadas na tentativa de se verificar a influência de tais fatores para a patogênese $e$ progressão de OMIM, principalmente no que concerne à ocorrência de infecção $^{12,13}$.

Inicialmente, a infecção era considerada como um fator secundário na progressão e estabelecimento de OMIM, porém, após a realização de algumas pesquisas envolvendo pacientes houve um restabelecimento da importância dos microrganismos para 0 desenvolvimento da doença ${ }^{5}$. Uma observação considerável nesses trabalhos é que, embora a maioria dos casos relatados de OMIM indique 0 desenvolvimento da lesão após 0 procedimento cirúrgico de exodontia, os dentes extraídos apresentavam ou doença periodontal ou periapical ${ }^{14-16}$. Além disso, estudos em animais foram conduzidos para se demonstrar que a inflamação e infecção, na presença de agentes antirreabsortivos sistêmicos, são suficientes para induzir a OMIM ${ }^{17-19}$. Portanto, secundária ou não, a participação dos microrganismos no desenvolvimento de OMIM é evidente, e o controle do processo infeccioso apresenta-se essencial para o manejo da patologia ${ }^{20}$; nesse sentido, torna-se essencial 0 estabelecimento do perfil microbiológico associado às lesões osteonecróticas.

Os primeiros estudos que avaliaram amostras ósseas necróticas humanas relacionadas à OMIM, analisaram aquelas induzidas por bisfosfonatos (OMIB) e puderam identificar a presença do gênero bacteriano Actinomyces ${ }^{21-30}$. Pesquisas subsequentes passaram a avaliar a possibilidade da existência de um biofilme complexo e específico sobre o osso exposto das lesões osteonecróticas $^{6,31,32}$. Apesar disso, ainda não há uma elucidação completa sobre as espécies de microrganismos associados ao desenvolvimento da OMIM, principalmente as induzidas por bisfosfonatos, 0 que permitiria um direcionamento terapêutico mais adequado durante o emprego dos agentes antimicrobianos locais e sistêmicos utilizados no controle da doença. O objetivo desta revisão, portanto, foi avaliar trabalhos que investigassem o perfil microbiológico bucal de pacientes portadores da osteonecrose maxilar induzida por bisfosfonatos (OMIB).

\section{Material e método}

Esta revisão de literatura baseou-se na busca por artigos científicos na base de dados do Medline-Pubmed (www.pubmed.com) datados de 2003 a 2015, e que apresentassem os seguintes descritores: "osteonecrosis", "jaw" e "microrganisms". No total, dezoito artigos que correlacionaram a uma análise microbiológica e a ocorrência da OMIB foram selecionados.

\section{Revisão de literatura}

O tratamento de pacientes com lesão estabelecida de osteonecrose maxilar induzida por medicamentos objetiva o controle da dor, da infecção e da progressão da necrose óssea. Dessa maneira, a prescrição de antibióticos sistêmicos é recomendada; no entanto, não há uma classe farmacológica específica e comprovadamente eficaz no controle e evolução da doença, pois se faz necessária uma melhor elucidação das espécies microbianas envolvidas ${ }^{5}$.

Em 1983, Happonem et al. ${ }^{21}$, identificaram a presença de Actinomyces israelli em pacientes com osteorradionecrose maxilar. A observação dessa espécie bacteriana em lesões de osteonecrose não induzidas 
por medicamentos justificou pesquisas que também visaram verificar a presença dessa bactéria em lesões de OMIB. Assim, a partir do primeiro relato da OMIB em $2003^{16}$, o gênero Actinomyces spp tem sido estudado e identificado em pacientes com a patologia ${ }^{21-23}$.

Hansen et al. ${ }^{22}$, através de análise histopatológica, observaram a presença de Actinomyces spp em todas as amostras de tecido ósseo necrótico de oito paciente com mieloma múltiplo e câncer de mama, e que desenvolveram OMIB devido ao uso de pamidronato, ácido zoledrônico ou ibandronato. Os autores ainda sugeriram uma relação entre a bactéria e a fisiopatologia da doença $^{22}$. Da mesma forma, outras pesquisas também reportaram, através da análise histopatológica, a presença do gênero bacteriano em lesões ósseas de pacientes com $\mathrm{OMIB}^{25-30}$, como no trabalho de Jacobsen et al. (2012) que relataram 46 amostras de lesões ósseas apresentando Actinomyces spp das 64 obtidas de pacientes com $\mathrm{OMIB}^{24}$.

Além da avaliação histopatológica, a utilização da técnica de cultura microbiológica associada foi utilizada em outros trabalhos que estudaram a microbiota presente em lesões de OMIB. Assim, Badros et al. ${ }^{6}$ avaliaram 20 amostras de lesões ósseas de pacientes com mieloma múltiplo, descrevendo a presença de microrganismos filamentosos compatíveis com Actinomyces spp em 7 deles. Nesse mesmo estudo, foram identificadas variadas espécies bacterianas pertencentes aos gêneros Peptostreptococcus spp, Streptococcus spp, Eikenella spp, Prevotella spp, Porphyromonas spp e Fusobacterium spp em 9 espécimes avaliados ${ }^{6}$.

De maneira semelhante, a pesquisa conduzida por Sedghizadeh et al. ${ }^{31}$, realizada com 4 pacientes com OMIB, sendo 2 diagnosticados com osteoporose, um com mieloma múltiplo e outro com câncer de próstata e tratados respectivamente com alendronato, zoledronato e pamidronato, identificou uma variedade de espécies microbianas, em especial, as bactérias dos gêneros Fusobacterium spp, Bacillus spp, Actinomyces spp, Staphylococcus spp, Streptococcus spp, Selenomonas spp e três variantes morfológicas de spirochetas $^{31}$. Além disso, após corte transversal dos espécimes, os autores descreveram a presença de bactérias em todas as superfícies internas do osso, indicando a presença de um biofilme bacteriano em cavidades ósseas mais profundas e não somente em superfícies expostas à cavidade oral contaminada.

Finalmente, O'Ryan \& Lo. ${ }^{32}$ avaliaram 12 amostras de lesões ósseas necróticas de pacientes que estavam em tratamento para osteoporose ou osteopenia utilizando bisfosfonatos por via oral (alendronato, ibandronato ou etidronato) e identificaram, por métodos histopatológicos e por cultura microbiológica, a presença de Actinomyces spp, Streptococcus spp, Prevotella spp, Klebsiella spp e Pseudomonas spp ${ }^{32}$.

Um perfil microbiológico específico e mais abrangente dos pacientes com OMIB foi obtido através de pesquisas mais recentes e que utilizaram técnicas de análise biomolecular. Dessa maneira, Sedghizadeh et $\mathrm{al}^{33}$ realizaram uma investigação metagenômica para identificação dos microrganismos presentes em salivas de cinco pacientes com câncer de próstata ou osteoporose e que desenvolveram OMIB com a utilização de alendronato (VO) ou ácido zoledrônico (IV $)^{33}$. Os autores identificaram que $99 \%$ das bactérias encontradas pertenciam aos Filos Proteobacteria (70\%), Firmicutes (26,9\%) e Actinobacteria (1,95\%), sendo que as principais encontradas eram dos gêneros Klebsiella e Serratia (Filo Proteobacteria) e Streptococcus e Bacillus (Filo Firmicutes).

De acordo com $\mathrm{Kos}^{34}$ a importância da caracterização microbiológica de bactérias planctônicas presentes nas 
salivas de pacientes com OMIB relaciona-se aos processos cirúrgicos que ocorrem na cavidade bucal, como as exodontias. Procedimentos como esses poderiam ser a porta de entrada para microrganismos colonizadores se instalarem em lesões ósseas maxilares de pacientes usuários de bisfosfonatos ${ }^{34}$.

No estudo de Ji et al. ${ }^{35}$, amostras de lesões de 20 pacientes com OMIB, sendo 10 utilizando antibióticos sistêmicos e 10 sem histórico recente e tampouco a utilização da medicação, foram submetidas às análises biomoleculares de eletroforese em gel de gradiente desnaturante (DGGE) e de sequenciamento. Os autores relataram nos pacientes usuários de antibióticos, uma alta incidência de bactérias do Filo Firmicutes, em sua maioria, as espécies Streptococcus intermedius, Lactobacillus gasseri, Mogibacterium timidum e Solobacterium moorei. Em contrapartida, pacientes sem uso de antibióticos apresentaram grandes quantidades de Parvimonas micra, Streptotoccus anginosus e Atopobium rimae ${ }^{35}$.

Wei et al ${ }^{36}$ realizaram um estudo com 12 indivíduos com câncer de diferentes etiologias e sem a utilização de antibióticos sistêmicos até o momento da coleta das amostras, sendo que 6 pacientes apresentavam OMIB (grupo teste) e 6 continham infecção no osso maxilar sem a lesão ou histórico de utilização de bisfosfonatos (grupo controle $)^{36}$. As amostras de osso necrótico foram submetidas à análise molecular através da extração de DNA (ácido desoxirribonucleico) seguida da reação em cadeia pela polimerase (PCR). Os autores identificaram a presença de bactérias pertencentes a sete Filos distintos em ambos os grupos experimentais:

Actinobacteria, Bacteroidetes, Firmicutes, Fusobacteria, Proteobacteria, Spirochaetes e TM7. O Filo Fusobacteria foi evidenciado no grupo controle enquanto o TM7 foi identificado apenas no grupo OMIB; além disso, as bactérias do Filo Firmicutes foram as mais prevalentes tanto no grupo controle $(61 \%)$ quanto no grupo OMIB (76\%). Algumas espécies foram predominantes nos pacientes com OMIB tais como Pseudoramibacter alactolyticos (14\%), Streptococcus mitis (12\%), Atopobium spp (9\%), Mogibacterium timidum (9\%), Bacteroidetes bacterium oral taxon $272(8 \%)$, enquanto no grupo controle predominaram Lactobacillus gasseri (11\%), Streptococcus mutans (6\%) e Fusobacterium nucleatum (9\%). O estudo também verificou uma predominância dos gêneros bacterianos Streptococcus spp (29\%), Eubacterium spp $(9 \%)$ e Pseudoramibacter spp (8\%) para os pacientes com OMIB, e dos gêneros Parvimonas spp (17\%), Streptococcus spp (15\%) e Fusobacterium spp (15\%) para os indivíduos sem a patologia ${ }^{36}$.

Por fim, Pushalkar et al. ${ }^{37}$ analisaram amostras de lesões em tecido mole associadas ao osso necrótico maxilar de 30 pacientes, sendo que 15 indivíduos apresentavam o diagnóstico de OMIB sem uso prévio de antibióticos por pelo menos 3 meses, 5 eram portadores de doença periodontal com uso prévio de bisfosfonatos, porém sem OMIB, e 10 eram portadores de doença periodontal sem histórico de utilização prévia de bisfosfonatos. Os autores selecionaram aleatoriamente 5 amostras de cada grupo para as análises biomoleculares (PCR) e identificaram, nos três grupos estudados, bactérias pertencentes aos filos Actinobacteria, Bacteroidetes, Firmicutes, Fusobacteria, Proteobacteria e TM7. O estudo ressaltou a prevalência do Filo Firmicutes em todos os grupos avaliados, porém, com maior evidência no grupo com OMIB $(71 \%)$. Nesse mesmo grupo, grande quantidade das espécies pertencentes ao Filo Fusobacteria foram observadas, enquanto que no grupo controle, destacaram-se aquelas provenientes do Filo TM7. No grupo OMIB predominaram os gêneros bacterianos Parvimonas spp $(18,3 \%)$, Fusobacterium spp (4\%), 
Eubacterium[11][G6] spp (3,1\%), Gemella spp (2,1\%), Leptotrichia spp $(1,7 \%)$ e Selenomonas spp (1\%), sendo que os gêneros Xanthomonas spp (3,8\%), Lachnospiraceae[G7] spp $(2,1 \%)$, Eubacterium[11][G1] spp (2,1\%), Bifidobacterium spp (2\%) e Bacterioides[G2] spp foram encontrados exclusivamente, e as espécies bacterianas Parvimonas micra, Streptococcus anginosus, Atopobium rimae, Peptostreptococcus stomatis e Eubacterium[11][G-6] nodatum ${ }^{37}$.

\section{Discussão}

A patogênese da osteonecrose maxilar induzida por medicamentos (OMIM) ainda precisa ser melhor elucidada. Muitos estudos apoiam a hipótese de que a infecção está diretamente relacionada com 0 desenvolvimento da lesão, desempenhando uma significante influência ${ }^{20}$. Sabe-se que a presença de inflamação ou infecção na cavidade bucal é um fator de risco para o desenvolvimento da OMIM e, atualmente, estudos clínicos e em modelos animais têm demonstrado que as duas condições são componentes importantes da patologia. Apesar disso, pouco está definido sobre as espécies microbianas que compõem o biofilme presente no osso exposto e por isso pesquisas têm sido conduzidas com o objetivo de elucidar essa questão ${ }^{5}$.

Os estudos que avaliaram biópsias de osso necrótico provenientes de indivíduos com a osteonecrose maxilar induzida por bisfosfonatos (OMIB) identificaram principalmente a presença do gênero bacteriano Actinomyces spp. Através de análises histopatológicas, os trabalhos de Hansen et al. ${ }^{22}$, Lee et al. ${ }^{26}$ e Arranz Caso et al. ${ }^{25}$ detectaram Actinomyces spp em todos os pacientes com OMIB estudados, demonstrando assim, uma associação entre a presença de bactérias desse gênero e a patologia envolvida ${ }^{22,25-27}$. No entanto, Jacobsen et al. ${ }^{24}$, que também utilizaram a histopatologia para a identificação dos microrganismos, observaram que Actinomyces spp não esteve presente nas lesões ósseas de todos os pacientes com OMIB avaliados, concluindo que outras espécies de microrganismos poderiam estar associadas à lesão ${ }^{28}$.

Além da análise histopatológica, Badros et al. ${ }^{6}$, Dannemman et al. ${ }^{38}$, e Wongchuensoontorn et al. ${ }^{39}$ utilizaram a cultura microbiológica para detecção dos microrganismos envolvidos com as lesões de OMIB e descreveram, além de Actinomyces spp, a presença dos gêneros Peptostreptococcus spp, Streptococcus spp, Enterococcus faecalis, Actinomyces israelii, Bacteroides fragilis e Bacteroides melaninogenicus. A partir dessa avaliação microbiológica através de uma metodologia um pouco mais abrangente, os estudos detectaram outros gêneros bacterianos anteriormente não descritos e que poderiam estar relacionados à lesão osteonecrótica. Em contrapartida, supõe-se que a caracterização de um perfil microbiológico de pacientes com OMIB não pode ser melhor elucidada nesses estudos devido às dificuldades das técnicas de análises histopatológica e de cultura microbiana, que incluem, as deficientes identificação e quantificação de espécies de microrganismos ${ }^{6,38-40}$.

Nesse sentido, as pesquisas que utilizaram análises de biologia molecular para a identificação microbiológica de lesões ósseas de pacientes com OMIB, puderam elucidar de maneira mais abrangente as diferentes espécies, gêneros e Filos que compõem o biofilme associado à lesão, além de quantificá-los.

Os trabalhos de Wei et al. ${ }^{36}$, Ji et al. ${ }^{35}$, Sedghizadeh et al. ${ }^{31}$ e Pushalkar et al. ${ }^{37}$ observaram, através de análises biomoleculares, uma variedade de microrganismos associados às lesões ósseas de OMIB que não somente Actinomyces spp. Bactérias pertencentes aos Filos Actinobacteria, Bacteroidetes, 
Firmicutes, Fusobacteria, Proteobacteria, Spirochaetes e TM7 estiveram presentes na maioria desses estudos e, apesar da diversidade encontrada, percebe-se que um perfil de microrganismos associados à OMIB parece existir. Assim, alguns gêneros bacterianos foram descritos tanto em maior frequência quanto em quantidade como Pseudoramibacter spp, Streptococcus spp, Parvimonas spp, Mogibacterium spp, Gemella spp, Eubacterium spp (Filo Firmicutes), Atopobium spp (Filo Actinobacteria), e Bacteroides spp (Filo Bacteroidetes). Embora a maioria das pesquisas encontradas relacionarem o gênero Actinomyces spp como o mais frequente nas lesões de OMIB, esses estudos se basearam em uma análise histopatológica que envolveu a identificação microbiana sem a quantificação das espécies ou gêneros. Além disso, espécies bacterianas difíceis de serem cultivadas possivelmente não foram detectadas nos testes histopatológicos ${ }^{36,33,37}$.

Portanto, a biologia molecular é a técnica mais adequada para a determinação de um perfil microbiológico dos pacientes com OMIB. Apesar do gênero Actinomyces spp ter sido o mais prevalente nos estudos analisados, mais pesquisas são necessárias para a quantificação e classificação dos principais microrganismos envolvidos na patologia, o que será de grande valia tanto para a elucidação de sua patogenia quanto para o direcionamento de sua terapêutica antimicrobiana.

\section{Conclusões}

A maioria dos estudos selecionados destacou a presença do gênero Actinomyces spp em lesões de pacientes com OMIB.

Houve uma variação, relacionada às técnicas metodológicas utilizadas na caracterização dos microrganismos, do perfil microbiológico dos pacientes portadores de lesões de OMIB.

Em estudos que utilizaram análises histopatológicas o gênero Actinomyces $s p p$ foi o principal descrito, enquanto àqueles que utilizaram análise biomolecular descreveram principalmente Pseudoramibacter spp, Streptococcus spp, Parvimonas spp, Mogibacterium spp, Gemella spp, Eubacterium spp (Filo Firmicutes), Atopobium spp (Filo Actinobacteria) e Bacteroides spp (Filo Bacteroidetes).

Mais estudos são necessários para a caracterização e quantificação dos principais microrganismos associados às lesões de OMIB. 
Referências

1. Drake MT, Clarke BL, Khosla S. Bisphosphonates: mechanism of action and role in clinical practice. Mayo Clinic proceedings. 2008 Sep;83(9):1032-45. DOI: $10.4065 / 83.9 .1032$.

2. Bilezikian JP. Osteonecrosis of the jaw-do bisphosphonates pose a risk? The New England journal of medicine. 2006 Nov 30;355(22):2278-81. DOI: 10.1056/NEJMp068157.

3. Marx RE. Pamidronate (Aredia) and zoledronate (Zometa) induced avascular necrosis of the jaws: a growing epidemic. Journal of oral and maxillofacial surgery : official journal of the American Association of Oral and Maxillofacial Surgeons. 2003 Sep;61(9):1115-7. DOI: https://doi.org/10.1016/S02782391(03)00720-1

4. Russell RG, Watts NB, Ebetino FH, Rogers MJ. Mechanisms of action of bisphosphonates: similarities and differences and their potential influence on clinical efficacy. Osteoporosis international : a journal established as result of cooperation between the European Foundation for Osteoporosis and the National Osteoporosis Foundation of the USA. 2008 Jun;19(6):733-59. DOI: 10.1007/s00198007-0540-8.

5. Ruggiero SL, Dodson TB, Fantasia J, Goodday R, Aghaloo T, Mehrotra B, et al. American Association of Oral and Maxillofacial Surgeons position paper on medication-related osteonecrosis of the jaw--2014 update. Journal of oral and maxillofacial surgery : official journal of the American Association of Oral and Maxillofacial Surgeons. 2014 Oct;72(10):1938-56.

DOI: https://doi.org/10.1016/j.joms.2015. 03.060

6. Badros A, Weikel D, Salama A, Goloubeva O, Schneider A, Rapoport A, et al. Osteonecrosis of the jaw in multiple myeloma patients: clinical features and risk factors. Journal of clinical oncology : official journal of the American Society of Clinical Oncology. 2006 Feb
20;24(6):945-52.

DOI:

10.1200/JCO.2005.04.2465.

7. Kumar V, Sinha RK. Bisphosphonate Related Osteonecrosis of the Jaw: An Update. Journal of maxillofacial and oral surgery. $2014 \quad$ Dec;13(4):386-93. DOI:10.1007/s12663-013-0564-X.

8. Ruggiero SL, Mehrotra B. Bisphosphonate-related osteonecrosis of the jaw: diagnosis, prevention, and management. Annual review of medicine. 2009;60:85-96.

DOI: 10.1146/annurev.med.60.063007.134350 .9. Diego R, D'Orto O, Pagani D, Agazzi A, Marzano U, Derada Troletti G, et al. Bisphosphonate-associated osteonecrosis of the jaws: a therapeutic dilemma. Oral surgery, oral medicine, oral pathology, oral radiology, and endodontics. 2007 Mar;103(3):e1-5. DOI: 10.1016/j.tripleo.2006.09.022.

10. Migliorati CA, Casiglia J, Epstein J, Jacobsen PL, Siegel MA, Woo SB. Managing the care of patients with bisphosphonate-associated

osteonecrosis: an American Academy of Oral Medicine position paper. Journal of the American Dental Association. 2005 Dec;136(12):1658-68.

11. Petropoulos VC, Balshi TJ, Wolfinger GJ, Balshi SF. Treatment of a Patient with Implant Failure and Jaw Osteonecrosis: Successful Retreatment Using Implants. The Journal of oral implantology. 2014 Mar 25. DOI: 10.1563/AAID-JOI-D-1300186.

12. Dimopoulos MA, Kastritis E, Bamia C, Melakopoulos I, Gika D, Roussou M, et al. Reduction of osteonecrosis of the jaw (ONJ) after implementation of preventive measures in patients with multiple myeloma treated with zoledronic acid. Annals of oncology : official journal of the European Society for Medical Oncology / ESMO. 2009 Jan;20(1):117-20. DOI: 10.1093/annonc/mdn554.

13. Hoff $A O$, Toth $B B$, Altundag $K$, Johnson $\mathrm{MM}$, Warneke $\mathrm{CL}$, $\mathrm{Hu} \mathrm{M}$, et al. Frequency and risk factors associated with osteonecrosis of the jaw in cancer patients treated with intravenous bisphosphonates. Journal of bone and mineral research : the official journal of 
the American Society for Bone and Mineral Research. 2008 Jun;23(6):82636. DOI: 10.1359/jbmr.080205.

14. Boonyapakorn T, Schirmer I, Reichart PA, Sturm I, Massenkeil G. Bisphosphonate-induced osteonecrosis of the jaws: prospective study of 80 patients with multiple myeloma and other malignancies. Oral oncology. 2008 Sep;44(9):857-69.

DOI: https://doi.org/10.1016/j.oraloncolog y.2007.11.012.

15. Marx RE, Sawatari $Y$, Fortin M, Broumand V. Bisphosphonate-induced exposed bone (osteonecrosis/ osteopetrosis) of the jaws: risk factors, recognition, prevention, and treatment. Journal of oral and maxillofacial surgery : official journal of the American Association of Oral and Maxillofacial Surgeons. 2005 Nov;63(11):1567-75. DOI: 10.1016/j.joms.2005.07.010.

16. Ficarra $G$, Beninati $F$, Rubino I, Vannucchi $A$, Longo $G$, Tonelli $P$, et al. Osteonecrosis of the jaws in periodontal patients with a history of bisphosphonates treatment. Journal of clinical periodontology. 2005 Nov;32(11):1123-8. DOI: 10.1111/j.1600051X.2005.00842.x.

17. Aguirre Jl, Akhter MP, Kimmel DB, Pingel JE, Williams $A$, Jorgensen $M$, et al. Oncologic doses of zoledronic acid induce osteonecrosis of the jaw-like lesions in rice rats (Oryzomys palustris) with periodontitis. Journal of bone and mineral research : the official journal of the American Society for Bone and Mineral Research. 2012 Oct;27(10):2130-43. DOI: 10.1002/jbmr.1669.

18. Kang B, Cheong S, Chaichanasakul T, Bezouglaia O, Atti E, Dry SM, et al. Periapical disease and bisphosphonates induce osteonecrosis of the jaws in mice. Journal of bone and mineral research : the official journal of the American Society for Bone and Mineral Research. 2013 Jul;28(7):1631-40. DOI: 10.1002/jbmr.1894.

19. Aghaloo TL, Kang B, Sung EC, Shoff M, Ronconi M, Gotcher JE, et al. Periodontal disease and bisphosphonates induce osteonecrosis of the jaws in the rat. Journal of bone and mineral research : the official journal of the American Society for Bone and Mineral Research. 2011 Aug;26(8):1871 82. DOI: 10.1002/jbmr.379.

20. Thumbigere-Math V, Sabino MC, Gopalakrishnan R, Huckabay S, Dudek AZ, Basu S, et al. Bisphosphonaterelated osteonecrosis of the jaw: clinical features, risk factors, management, and treatment outcomes of 26 patients. Journal of oral and maxillofacial surgery : official journal of the American Association of Oral and Maxillofacial Surgeons. 2009 Sep;67(9):1904-13. DOI: 10.1016/j.joms.2009.04.051.

21. Happonen RP, Viander M, Pelliniemi L, Aitasalo K. Actinomyces israelii in osteoradionecrosis of the jaws. Histopathologic and immunocytochemical study of five cases. Oral surgery, oral medicine, and oral pathology. 1983 Jun;55(6):580-8.

22. Hansen T, Kunkel M, Weber A, James Kirkpatrick C. Osteonecrosis of the jaws in patients treated with bisphosphonates - histomorphologic analysis in comparison with infected osteoradionecrosis. Journal of oral pathology \& medicine : official publication of the International Association of Oral Pathologists and the American Academy of Oral Pathology. 2006 Mar;35(3):15560. DOI: $10.1111 / \mathrm{j} .1600$ 0714.2006.00391.x.

23. Naik NH, Russo TA. Bisphosphonaterelated osteonecrosis of the jaw: the role of actinomyces. Clinical infectious diseases : an official publication of the Infectious Diseases Society of America. 2009 Dec 1;49(11):1729-32. DOI: https://doi.org/10.1086/648075.

24. Jacobsen C, Metzler P, Obwegeser JA, Zemann W, Graetz KW. Osteopathology of the jaw associated with bone resorption inhibitors: what have we learned in the last 8 years? Swiss medical weekly. 2012;142:w13605. DOI: https://doi.org/10.4414/smw.2012.13605.

25. Arranz Caso JA, Flores Ballester E, Ngo Pombe S, Lopez Pizarro V, DominguezMompello JL, Restoy Lozano A. 
[Bisphosphonate related osteonecrosis of the jaw and infection with Actinomyces]. Medicina clinica. 2012 Dec 15;139(15):676-80. DOI: 10.1016/j.medcli.2012.05.039.

26. Lee CY, Pien FD, Suzuki JB. Identification and treatment of bisphosphonate-associated actinomycotic osteonecrosis of the jaws. Implant dentistry. 2011 Oct;20(5):331-6. DOI: 10.1097/ID.0b013e3182310f03.

27. Hansen T, Kunkel M, Springer E, Walter C, Weber A, Siegel E, et al. Actinomycosis of the jaws-histopathological study of 45 patients shows significant involvement in bisphosphonate-associated

osteonecrosis and infected osteoradionecrosis. Virchows Archiv : an international journal of pathology. 2007 Dec;451(6):1009-17.

DOI: 10.1007/s00428-007-0516-2.

28. Merigo E, Manfredi M, Meleti M, Corradi $D$, Vescovi P. Jaw bone necrosis without previous dental extractions associated with the use of bisphosphonates (pamidronate and zoledronate): a fourcase report. Journal of oral pathology \& medicine : official publication of the International Association of Oral Pathologists and the American Academy of Oral Pathology. 2005 Nov;34(10):6137.

DOI:10.1111/j.1600-

0714.2005.00351.x.

29. Lazarovici TS, Yahalom R, Taicher S, Elad S, Hardan I, Yarom N. Bisphosphonate-related osteonecrosis of the jaws: a single-center study of 101 patients. Journal of oral and maxillofacial surgery : official journal of the American Association of Oral and Maxillofacial Surgeons. 2009 Apr;67(4):850-5. DOI: 10.1016/j.joms.2008.11.015.

30. Senel FC, Saracoglu Tekin U, Durmus A, Bagis B. Severe osteomyelitis of the mandible associated with the use of nonnitrogen-containing bisphosphonate (disodium clodronate): report of a case. Journal of oral and maxillofacial surgery : official journal of the American Association of Oral and Maxillofacial Surgeons. 2007 Mar;65(3):562-5. DOI: 10.1016/j.joms.2006.10.043.
31. Sedghizadeh PP, Kumar SK, Gorur A, Schaudinn C, Shuler CF, Costerton JW. Identification of microbial biofilms in osteonecrosis of the jaws secondary to bisphosphonate therapy. Journal of oral and maxillofacial surgery : official journal of the American Association of Oral and Maxillofacial Surgeons. 2008 Apr;66(4):767-75.

DOI: 10.1016/j.joms.2007.11.035.

32. O'Ryan FS, Lo JC. Bisphosphonaterelated osteonecrosis of the jaw in patients with oral bisphosphonate exposure: clinical course and outcomes. Journal of oral and maxillofacial surgery : official journal of the American Association of Oral and Maxillofacial Surgeons. 2012 Aug;70(8):1844-53. DOI:10.1016/j.joms.2011.08.033.

33. Sedghizadeh PP, Yooseph S, Fadrosh DW, Zeigler-Allen $L$, Thiagarajan $M$, Salek $\mathrm{H}$, et al. Metagenomic investigation of microbes and viruses in patients with jaw osteonecrosis associated with bisphosphonate therapy. Oral surgery, oral medicine, oral pathology and oral radiology. 2012 Dec;114(6):764-70. DOI: 10.1016/j.0000.2012.08.444

34. Kos M. WITHDRAWN: Bisphosphonates promote jaw osteonecrosis through facilitating bacterial colonisation. Medical hypotheses. 2011 Aug;77(2):214-5. DOI: https://doi.org/10.1016/j.bihy.2008.08.00 4.

35. Ji X, Pushalkar S, Li Y, Glickman R, Fleisher K, Saxena D. Antibiotic effects on bacterial profile in osteonecrosis of the jaw. Oral Diseases, vol.18, n.1, p.8595, 2012. DOI: 10.1111/j.16010825.2011.01848.x.

36. Wei X, Pushalkar S, Estilo C, Wong C, Farooki A, Fornier M, et al. Molecular profiling of oral microbiota in jawbone samples of bisphosphonate-related osteonecrosis of the jaw. Oral diseases. 2012 Sep;18(6):602-12. DOI:10.1111/j.1601-0825.2012.01916.x.

37. Pushalkar S, Li, X, Kurago Z, Ramanathapuram LV, Matsumura $S$, Fleisher KE, Glickman R, Yan, W, Li Y, Saxena D. Oral microbiota and host innate immune response in bisphosphonate-related osteonecrosis of 
the jaw. International Journal of Oral Sciences., vol.6, n.4, p.219-226, 2014. DOI: DOI: 10.1038/ijos.2014.46.

38. Dannemann C, Zwahlen R, Grätz KW. Clinical experiences with bisphosphonate induced osteochemonecrosis of the jaws. Swiss Medical Weekly., vol.136, p.504509, 2006. DOI: 2006/31/smw-11431.

39. Wongchuensoontorn C, Liebehenschel N, Wagner K, Fakler O, Gutwald R, Schemlzeisen $R$, et al. Pathological fractures in patients caused by bisphosphonate-related osteonecrosis of the jaws: report of 3 cases. Journal of Oral and Maxillofacial Surgery., vol.67, p.13111316, 2009.2 DOI: : https://doi.org/10.1016/j.joms.2008.12.03 0 .

40. Woo PC, Lau SK, Teng JL, Tse H, Yuen $\mathrm{KY}$. Then and now: use of $16 \mathrm{~S}$ rDNA gene sequencing for bacterial identification and discovery of novel bacteria in clinical microbiology laboratories. Clinical microbiology and infection : the official publication of the European Society of Clinical Microbiology and Infectious Diseases. 2008 Oct;14(10):908-34. DOI: 10.1111/j.1469-0691.2008.02070.x. 